\title{
High mobility organic semiconductor for constructing high efficiency carbon nitride heterojunction photocatalysts
}

Yan Yi, Siyu Wang, Hantang Zhang, Jie Liu, Xiuqiang Lu, Lang Jiang, Chengji Sui, Hai Fan, Shiyun Ai and Jianwu Sun

The self-archived postprint version of this journal article is available at Linköping University Institutional Repository (DiVA):

http://urn.kb.se/resolve?urn=urn:nbn:se:liu:diva-172605

N.B.: When citing this work, cite the original publication.

Yi, Y., Wang, S., Zhang, H., Liu, J., Lu, X., Jiang, L., Sui, C., Fan, H., Ai, S., Sun, J., (2020), High mobility organic semiconductor for constructing high efficiency carbon nitride heterojunction photocatalysts, Journal of Materials Chemistry C, 8(48), 17157-17161.

https://doi.org/10.1039/dotc05123c

Original publication available at:

https://doi.org/10.1039/dotc05123c

Copyright: Royal Society of Chemistry

http://www.rsc.org/ 


\section{High mobility organic semiconductor for constructing high efficiency carbon nitride heterojunction photocatalysts}

Received 00th January 20xx, Accepted 00th January 20xx

\author{
Yan Yi, ${ }^{\text {ta }}$ Siyu Wang, ${ }^{\text {ta }}$ Hantang Zhang, ${ }^{* a}$ Jie Liu, ${ }^{c}$ Xiuqiang Lu, ${ }^{d}$ Lang Jiang,,${ }^{c}$ Chengji Sui, ${ }^{a}$ Hai Fan, ${ }^{a}$ \\ Shiyun $\mathrm{Ai}^{* a}$ and Jianwu Sun*b
}

\begin{abstract}
Graphitic carbon nitride (CN) has attracted worldwide attention due to its low-cost, environmental friendliness and tunable structures. However, $\mathrm{CN}$ photocatalyst still suffers from low charge separation efficiency issue. It is known that mobility is one of the most important factors dominating the charge separation and transport. This work reports a new strategy to address low charge separation issue by coupling high-mobility organic semiconductor and $\mathrm{CN}$. Copper phthalocyanine (CuPc), a classical small molecule organic semiconductor which possesses high mobility, outstanding stability and strong visible-light absorption, was selected to construct $\mathrm{CN} / \mathrm{CuPc}$ heterojunctions. The $\mathrm{CN} / \mathrm{CuPc}$ heterojunctions showed a high hydrogen evolution (PHE) rate of $9.0 \mathrm{mmol} \mathrm{g}^{-1} \mathrm{~h}^{-1}$ and apparent quantum yield (AQY) of 3.99\% (420 nm) and $1.31 \%$ $(600 \mathrm{~nm})$. This work demonstrates the application prospects of high-mobility organic semiconductors in heterojunction photocatalysis.
\end{abstract}

Visible-light induced photocatalytic hydrogen evolution (PHE) has attracted tremendous research interests since it provides a sustainable approach to address the forthcoming worldwide energy and environmental crisis. $^{1}$ Efficient visible-light absorption, high efficiency and favorable stability are essential requirements for PHE photocatalysts. Besides, in terms of potential commercial application, photocatalysts should also be low-cost and eco-friendly. Recently, graphitic carbon nitride (CN), as a metal-free and visible-light responsive organic photocatalyst, has grabbed intense attentions due to its fascinating features, such as appropriate energy band positions for PHE, high physicochemical stability, reasonable cost, nontoxicity and easy preparation. ${ }^{2-6}$ However, pristine $\mathrm{CN}$ suffers from deficient visible-light absorption and rapid recombination of photogenerated charges, resulting in moderate photocatalytic efficiency. Rational construction of nano-composites heterojunction has become an effective way to overcome these issues, since it can not only inhibit the hole- electron pairs recombination but also reinforce visible-light utilization. ${ }^{4}$ Recently, organic semiconductors (OSCs) have been regarded as an immense potential candidate to form efficient $\mathrm{CN}$ based organic-organic surface heterojunctions. ${ }^{7-23}$ Thanks to the unique structural tunability of organic molecules, the band position and visible-light absorption capacity of CN/OSCs heterojunctions could be conveniently regulated to enhance the exciton separation efficiency at the interface and broaden the light absorption of the system.

Basically, the reaction processes of PHE can be divided into three steps, namely light absorption, charge transport and surface reaction. Tremendous efforts have been made to improve the light absorption and surface reaction processes, such as nanostructure design and molecular engineering. ${ }^{2}, 24$ However, little attention has been paid to the mobility of OSCS, which has an important influence on the recombination during the carrier transport, and therefore also affects the activity of the photocatalyst. The faster the carriers migrate, the lower the probability of the hole-electron recombination would be. Herein, copper phthalocyanine (CUPc), a classical OSC which possesses high mobility as well as excellent stability and efficient visible-light absorption, ${ }^{25-27}$ was used to construct $\mathrm{CN} / \mathrm{CuPc}$ pure organic heterojunctions with $\mathrm{CN}$ nanosheets through $\pi-\pi$ interaction. The heterojunction photocatalysts exhibit extended visible-light absorption and promoted charge transfer. As a result, the PHE rate is significantly increased compared with pure $\mathrm{CN}$, which is also higher than that of the previously reported similar $\mathrm{CN}$ based organic heterojunction system that possesses lower mobility, ${ }^{28}$ indicating the importance of mobility for the performance of organic

College of Chemistry and Material Science \& College of Resources and

Environment, Shandong Agriculture University, TaiAn 271018, China. E-mail: htzhang@sdau.edu.cn, ashiy@sdau.edu.cn

b. Department of Physics, Chemistry and Biology (IFM), Linköping University, Linköping SE-58183, Sweden. E-mail: jianwu.sun@liu.se

Beijing National Laboratory for Molecular Sciences, Key Laboratory of Organic

Solids, Institute of Chemistry, Chinese Academy of Sciences, Beijing 100190,

China.

d. School of Ocean Science and Biochemistry Engineering, Fuqing Branch of Fujian

Normal University, Fuqing 350300, China.

+ Equal contribution.

Electronic Supplementary Information (ESI) available: [Experimental section and characterization]. See DOI: 10.1039/x0xx00000x 


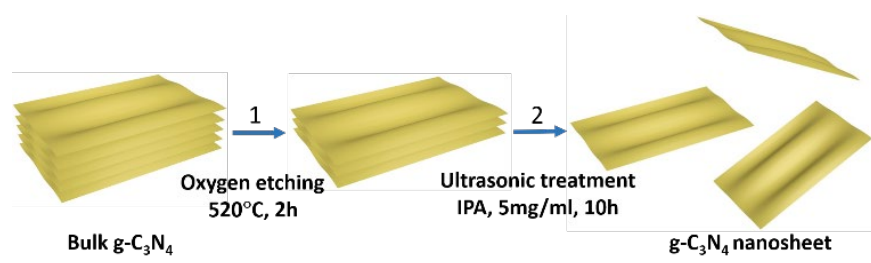

Scheme 1 Schematic of the two-step method for the exfoliation of bulk $\mathrm{CN}$ to $\mathrm{CN}$ nanosheets.

photocatalysts. To the best of our knowledge, this is the first report about the application of CuPc in organic heterojunction photocatalysts for PHE.

Bulk $\mathrm{CN}$ was fabricated by thermal condensation of urea at a temperature of $550^{\circ} \mathrm{C}$ for $4 \mathrm{~h}$. Then a two-step method combining secondary calcination and liquid exfoliation was adopted to obtain $\mathrm{CN}$ nanosheet, as shown in Scheme 1. Firstly, the thickness of bulk $\mathrm{CN}$ was decreased primarily through a secondary calcination in air. This step which is also known as thermal oxygen etching can break hydrogen bonds between $\mathrm{CN}$ layers through gradual oxidation. ${ }^{29} \mathrm{Fig}$. S2 shows the nitrogen absorption-desorption isotherms of bulk $\mathrm{CN}$ and $\mathrm{CN}$ after the secondary calcination. As derived from the isotherms, the specific surface area of $\mathrm{CN}$ is increased from $140.8 \mathrm{~m}^{2} / \mathrm{g}$ to 239.7 $\mathrm{m}^{2} / \mathrm{g}$ after the calcination. Meanwhile, the pore volume is increased from $0.61 \mathrm{~cm}^{3} / \mathrm{g}$ to $0.93 \mathrm{~cm}^{3} / \mathrm{g}$. These data verified the validity of this thermal treatment. Secondly, the obtained $\mathrm{CN}$ after secondary calcination was exfoliated into nanosheet via high-power ultrasonic processing in isopropanol for $10 \mathrm{~h} . \mathrm{X}$ ray diffraction (XRD) was conducted for $\mathrm{CN}$ before and after sonication (Fig. S3). The position of (002) peak, which originates from the interlayer stacking of $\mathrm{CN}$ nanosheet, shifts from $27.89^{\circ}$ to $27.67^{\circ}$, indicating an expanded interlayer distance after sonication. Simultaneously, the full width at half maximum (FWHM) became narrowed, manifesting improved crystallinity of $\mathrm{CN}$ which is caused by the better arrangement. The preparation of $\mathrm{CN}$ nanosheets by the two-step method was confirmed by the SEM images, as depicted in Fig. 1a and b. After two steps of exfoliation, large-sized and thick $\mathrm{CN}$ becomes small and thin nanosheets. The thickness of the ultrasonic treated $\mathrm{CN}$ nanosheets was determined by AFM (Fig. S4). The results showed that the $\mathrm{CN}$ nanosheet exhibited an ultrathin thickness of $\sim 1.9 \mathrm{~nm}$.

Considering the large conjugated structure of both $\mathrm{CN}$ and CuPc, the strong $\pi-\pi$ interaction could facilitate the construction of heterojunction. $\mathrm{CN} / \mathrm{CuPc}$ heterojunction photocatalysts with different ratios of CuPc (0 wt.\%, 3 wt.\%, 5 wt.\%, 7 wt.\%, 10 wt.\%) were prepared through a simple ultrasonic blending method. The typical TEM image of CN/CuPc (Fig. 1c, 7 wt.\% CuPc) illustrates that the heterojunction maintains the nanosheet morphology of CN. TEM-assisted elemental mappings were performed to prove the existence of CuPc in the composite, as shown in Fig. $1 \mathrm{~d}$ and Fig. S5. The Cu-k mapping indicates that CuPc is uniformly dispersed on $\mathrm{CN}$ nanosheets, and the heterojunction structure remains stable even after a series of sample preparation processes.
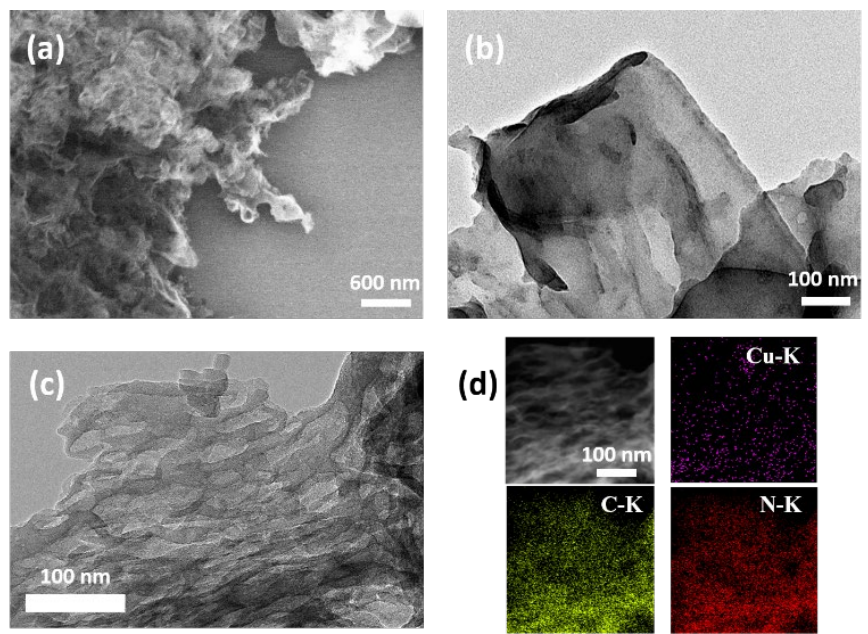

(d)

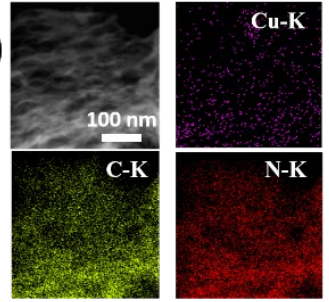

Fig. 1 SEM images of (a) bulk CN and (b) CN nanosheet obtained by the two-step method. (c) TEM image of $\mathrm{CN} / \mathrm{CuPc}$ (7 wt.\%) composite. (d) Electron image and corresponding TEM-assisted elemental mapping of $\mathrm{Cu}, \mathrm{C}$ and $\mathrm{N}$ of $\mathrm{CN} / \mathrm{CuPc}$ (7 wt.\%) composite.

The absorption spectrum of the heterojunction was measured by UV-vis diffuse reflectance spectroscopy (DRS), as shown in Fig. 2a. As can be seen, pristine $\mathrm{CN}$ has an absorption edge of $463 \mathrm{~nm}$, corresponding to an optical band gap about $2.68 \mathrm{eV}$ (Fig. 2b), which is slightly lower than some previously reported values. ${ }^{29-30}$ Considering that the $\mathrm{CN}$ fabrication and the initial exfoliation are carried out under air, it can be inferred that this may be caused by the $\mathrm{N}$ vacancy defects introduced unintentionally. ${ }^{31}$ While CuPc shows strong characteristic Qband absorption ranging from $500 \mathrm{~nm}$ to $800 \mathrm{~nm}$. Consequently, the CN/CuPc composites exhibit much broader absorption combining the features of $\mathrm{CN}$ and $\mathrm{CuPc}$, rendering the heterojunction with the ability to absorb almost the entire range of visible-light which is essential to improve the photocatalytic activity. Furthermore, the Q-band absorption of CuPc in CN/CuPc shows obvious red-shift compared with that of the pure $\mathrm{CuPc}$, which is an evidence suggesting $\pi-\pi$ interaction between $\mathrm{CN}$ and $\mathrm{CuPc}$. The interactions between $\mathrm{CN}$ and $\mathrm{CuPC}$ were further confirmed by the Fourier transform infrared spectroscopy (FTIR) and X-ray photoelectron spectroscopy (XPS). As depicted in Fig. 2c, the pristine $\mathrm{CN}$ shows typical FTIR absorption spectra. The peaks from $1200-1600 \mathrm{~cm}^{-1}$ correspond to the stretching vibration of $\mathrm{C}-\mathrm{N}$ heterocycles in $\mathrm{CN}$, and peak at $811 \mathrm{~cm}^{-1}$ represents the bending vibration of heptazine units. As the proportion of CuPc increases, the $\mathrm{CN}$ characteristic peaks become weak gradually, indicating the existence of noncovalent intermolecular interactions. ${ }^{7} \mathrm{Fig} .2 \mathrm{~d}$ shows the $\mathrm{Cu} 2 \mathrm{p}$ XPS spectra of pure CuPc and CN/CuPc (7 wt.\% CuPc). The binding energy of $\mathrm{Cu} 2 \mathrm{p}_{3 / 2}$ for $\mathrm{CN} / \mathrm{CuPc}$ decreases by $0.3 \mathrm{eV}$ compared with that of pure CuPc. Similar phenomenon is also observed for $\mathrm{Cu} 2 \mathrm{p}_{1 / 2}$ peak. Since $\mathrm{CN}$ is a nitrogen-rich material, the decrease of $\mathrm{Cu} 2 \mathrm{p}$ binding energy may be due to the enhancement of $\mathrm{Cu}-\mathrm{N}$ interaction, which leads to an increase in the outer electron density of $\mathrm{Cu}$ and thus enhances the electronic shielding effect. To prove this inference, $N$ 1s XPS spectra of $\mathrm{CN}$ and $\mathrm{CN} / \mathrm{CuPc}$ were conducted (Fig. S6). The $\mathrm{N}$ 1s 
(a)

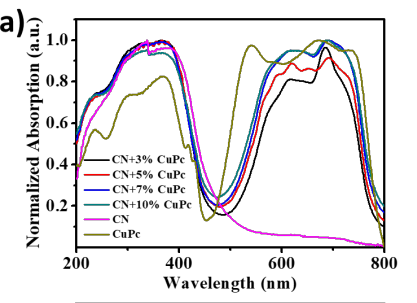

(c)

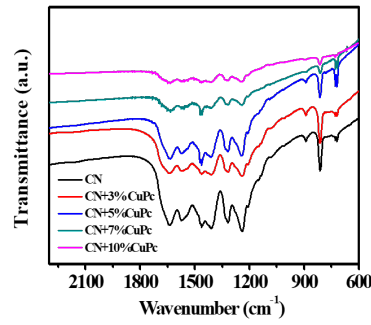

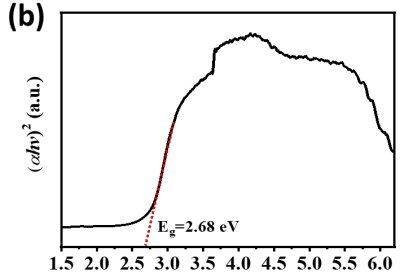

(d)

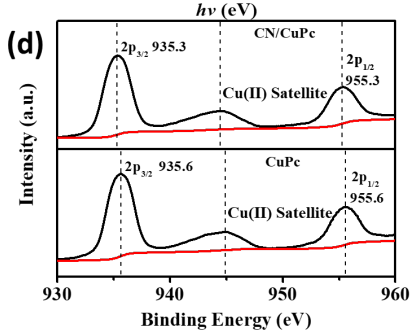

Fig. 2 (a) DRS of $\mathrm{CN}$ and $\mathrm{CN} / \mathrm{CuPc}$ heterojunctions. (b) Tauc plot derived from the DRS of CN. (c) FTIR of $\mathrm{CN}$ and CN/CuPc heterojunctions. The weight of different samples is the same ( $3 \mathrm{mg}$ ). (d) $\mathrm{Cu} 2 \mathrm{p}$ XPS of $\mathrm{CN}$ and $\mathrm{CN} / \mathrm{CuPc}$ (7 wt.\%) heterojunctions.

binding energy in the composites shows obvious increase compared with that of $\mathrm{CN}$, corresponding to the decrease of $\mathrm{Cu}$ $2 p$ binding energy.

To explore the photocatalytic activities of the CN/CuPc heterojunctions, the PHE rates of $\mathrm{CN}$ loaded with different ratios of CuPc were measured in the presence of triethanolamine (TEOA) as the hole sacrificial agent and without any other cocatalyst (Fig. S7 and Table S1). A 300 W Xenon lamp is used as a light source. A total of $20 \mathrm{mg}$ hybrids was used as the photocatalyst. The pristine $\mathrm{CN}$ nanosheets show low PHE rate of $15.2 \mu \mathrm{mol} \mathrm{g}^{-1} \mathrm{~h}^{-1}$. Initially, as the ratio of CuPc increases, the PHE rate of the heterojunction increase gradually, reaching the maximum rate of $71.2 \mu \mathrm{mol} \mathrm{g}{ }^{-1} \mathrm{~h}^{-1}$ at $7 \mathrm{wt} . \%$. However, further increasing the content of CuPc leads to a decrease of the PHE rate. This phenomenon is not exceptional since excessive CuPc may obscure the active site. ${ }^{12}$ In this case, the optimal ratio of $\mathrm{CuPc}$ is determined to be $7 \%$, under which the enhancement of visible-light absorption and the maintenance of the effective active sites could reach a balance. X-ray diffraction (XRD) of the $\mathrm{CN} / \mathrm{CuPc}$ before and after the reaction were perform to examine the stability of the mixture, as shown in Fig. S8. The XRD spectrum before the reaction shows a simple summation of the two components and remains basically the same after the reaction, indicating that CuPc has not been stripped off after a long time of reaction.

The photocatalytic activity of the heterojunction system could be further improved by adding Pt cocatalyst, as shown in Fig. 3. $5 \mathrm{wt} . \% \mathrm{Pt}$ was loaded onto the catalysts surface through the photodeposition of $\mathrm{H}_{2} \mathrm{PtCl}_{6} \cdot 6 \mathrm{H}_{2} \mathrm{O}$. Pure $\mathrm{CN}$ nanosheet with Pt cocatalyst exhibits a PHE rate of $3.4 \mathrm{mmol} \mathrm{g}^{-1} \mathrm{~h}^{-1}$, while the CN/CuPc heterojunction with 7 wt.\% CuPc shows a much higher rate of $9.0 \mathrm{mmol} \mathrm{g}^{-1} \mathrm{~h}^{-1}$ (Fig. 3a), which is also higher than that of the previously reported CN/VOPc system with lower mobility. ${ }^{28}$ These results demonstrate the advantages of highmobility organic semiconductors in improving the $\mathrm{CN}$ based $\mathrm{PHE}$ photocatalysts. The stability of the $\mathrm{CN} / \mathrm{CuPc}$ was also

(a)
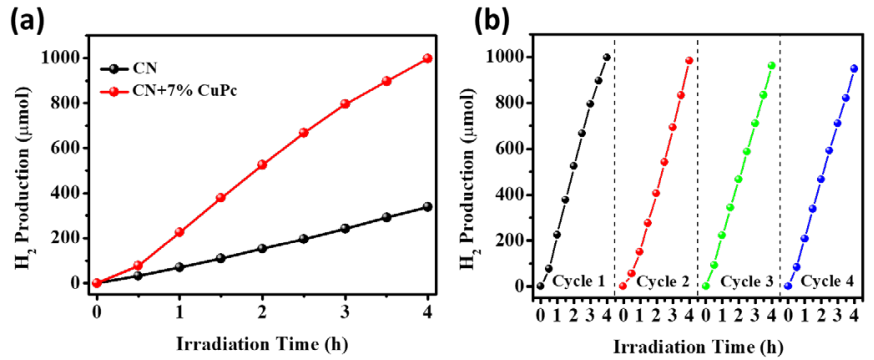

Fig. 3 (a) PHE results of $\mathrm{CN}$ and $\mathrm{CN} / \mathrm{CuPc}(7 \mathrm{wt} . \%$ ) heterojunction, measured under the irradiation of a $300 \mathrm{~W}$ Xenon lamp. TEOA is used as the hole sacrificial agent. 5 wt.\% Pt is used as cocatalyst. (b) Cycling test of the CN/CuPc (7 wt.\%) heterojunctions.

investigated by the cycling test (Fig. 3b). The photocatalytic activity of the composite shows no significant decline after four cycles of irradiation.

To further study the photocatalytic mechanism, it is essential to figure out the electronic interactions between $\mathrm{CN}$ and $\mathrm{CuPc}$. The band structure of $\mathrm{CN}$ and CuPc were determined by the DRS and Mott-Schottky analysis, as depicted in Fig. 4. The optical gap of CuPc is calculated as $1.40 \mathrm{eV}$ according to the DRS (Fig. 4a and $4 \mathrm{~b})$. The flat band position $\left(\mathrm{E}_{\mathrm{fb}}\right)$ of $\mathrm{CN}$ and CuPc can be obtained from the Mott-Schottky curves (Fig. $4 \mathrm{c}$ and $4 \mathrm{~d}$ ). For $\mathrm{CN}$, the positive slope indicates it is a typical $\mathrm{N}$-type semiconductor, and the $\mathrm{E}_{\mathrm{fb}}(-0.37 \mathrm{~V}$ vs $\mathrm{RHE})$ can be approximated as the conduction band $\left(\mathrm{E}_{\mathrm{C}}\right)$. For $\mathrm{CuPc}$, the negative slope indicates it is a P-type semiconductor and the $\mathrm{E}_{\mathrm{fb}}(0.88 \mathrm{~V}$ vs $\mathrm{RHE})$ can be approximated as the valence band $\left(E_{v}\right)$. Thus, straddled band alignment (Type II ) which could facilitate the charge transfer is formed, as shown in Fig. 5a. To verify the charge transfer interaction between the two components, photoluminescence (PL), photoresponse and electrochemical impedance spectroscopy (EIS) of $\mathrm{CN}$ and $\mathrm{CN} / \mathrm{CuPc}$ were conducted and compared, as shown in Fig. 5b-d. Pure CN shows strong emission at $469 \mathrm{~nm}$ under an excitation wavelength of $335 \mathrm{~nm}$ (Fig. 5b). The PL
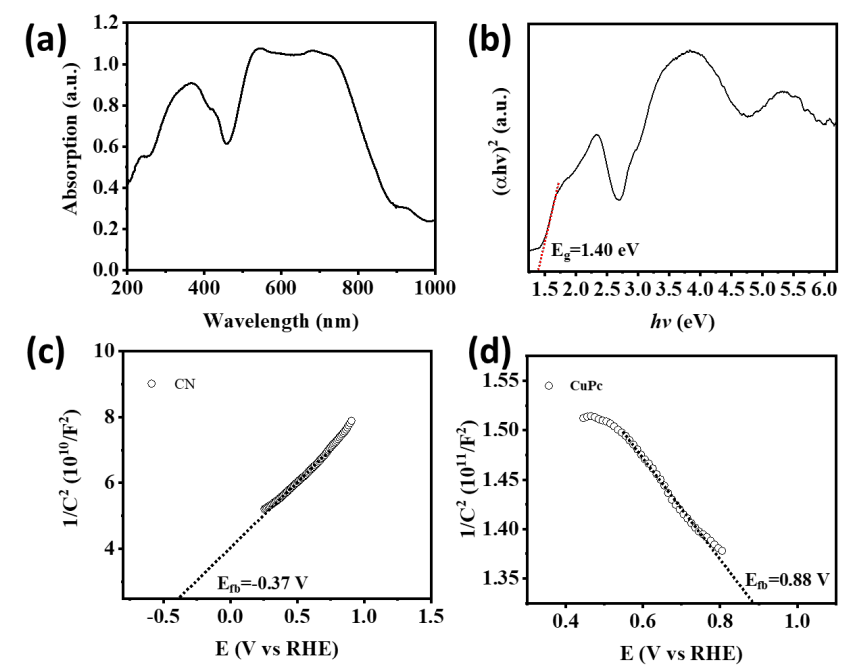

Fig. 4 (a) DRS of CuPc and (b) corresponding Tacu plots. Mott-Schottky plot of (c) CN and (d) CuPc. 
(a)
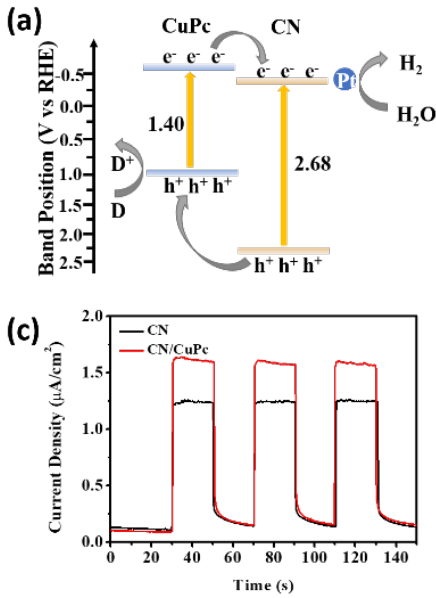

(b)

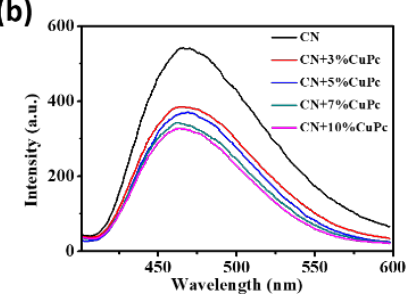

(d)

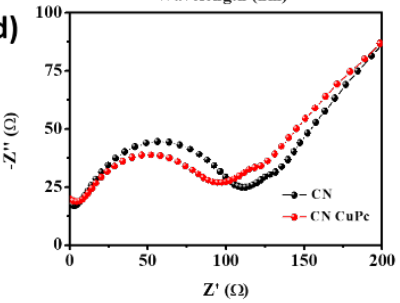

Fig. 5 (a) Schematic of the photocatalytic mechanism of CN/CuPc heterojunction. (b) $\mathrm{PL}$ of $\mathrm{CN}$ and $\mathrm{CN} / \mathrm{CuPc}$ heterojunctions under an excitation wavelength of $335 \mathrm{~nm}$. (c) Photocurrent responses of $\mathrm{CN}$ and $\mathrm{CN} / \mathrm{CuPc}$ (7 wt.\%) heterojunctions. $0.5 \mathrm{M} \mathrm{Na}_{2} \mathrm{SO}_{4}$ solution was used as the electrolyte. $2 \mathrm{mg} / \mathrm{ml} \mathrm{CN} / \mathrm{CuPc}$ isopropanol suspension was dripped onto the ITO electrode and baked dry with an infrared lamp. A 300w Xenon lamp was used as the light source. (d) EIS of CN and $\mathrm{CN} / \mathrm{CuPc}(7 \mathrm{wt} . \%)$ heterojunctions. $5 \mathrm{mM}\left[\mathrm{Fe}(\mathrm{CN})_{6}\right]^{3-/ 4-}(1: 1)$ solution containing $0.1 \mathrm{M} \mathrm{KCl}$ was used as the electrolyte. $2 \mathrm{mg} / \mathrm{ml} \mathrm{CN} / \mathrm{CuPc}$ isopropanol suspension was dripped onto the glassy carbon electrode and baked dry with an infrared lamp. ESI was tested using a three-electrode system.

intensity decreases with the increase of CuPc content, indicating that the recombination of the photogenerated electron-hole pairs are effectively suppressed in CN/CuPc heterojunction. Fig. $5 \mathrm{c}$ exhibits the photocurrent responses of $\mathrm{CN}$ and $\mathrm{CN} / \mathrm{CuPc}$ (7 wt.\% CuPc). Compared to $\mathrm{CN}$, higher photocurrent is obtained for $\mathrm{CN} / \mathrm{CuPc}$, which can be rationalized by the broader visible-light absorption and efficient charge transfer between $\mathrm{CN}$ and CuPc. To confirm the charge transfer process at the heterojunction interface, EIS of $\mathrm{CN}$ and $\mathrm{CN} / \mathrm{CuPc}$ (7 wt.\% CuPc) were also performed, the Nyquist plots are depicted in Fig. $5 \mathrm{~d}$. The semicircle in the high frequency region represents the charge transfer process at the electrode, and the arc radius indicates the charge transfer resistance $\left(R_{c t}\right)$. It is obvious that the CN/CuPc possesses a smaller radius and thus smaller $R_{c t}$, which means more effective charge transfer and migration for the heterojunction photocatalyst.

The photocatalytic mechanism can be further confirmed by PHE testing irradiated at monochromatic light. As shown in Fig. S9a, Under the irradiation of $600 \mathrm{~nm}$ monochromatic light beyond the $\mathrm{CN}$ absorption range, the entire system showed a PHE rate of $1.49 \mathrm{mmol} \mathrm{g}^{-1} \mathrm{~h}^{-1}$, corresponding to apparent quantum yield ( $A Q Y$ ) of $1.31 \%$. This means that there is effective charge transfer between CUPc and CN. The CN/CuPc hybrid under irradiation of $420 \mathrm{~nm}$ monochromatic for PHE was also measured (Fig. S9b), exhibiting a high AQY of $3.99 \%$. Furthermore, PHE testing of pure CuPc is also performed to determine which component the hydrogen production capacity is mainly derived from, as depicted in Fig. S10. Using Xenon lamp as the light source, pristine CuPc without Pt loaded has no hydrogen production capacity. After loading $5 \% \mathrm{Pt}$, CuPc shows weak PHE performance with a hydrogen production rate of 29.3 $\mu \mathrm{mol} \mathrm{g}^{-1} \mathrm{~h}^{-1}$, far below the value of the CN/CuPc system. Therefore, it can be inferred that the main sites of PHE reaction are located on the surface of $\mathrm{CN}$. The role of CuPc in the system is not only to broaden the visible light absorption but also to enhance the charge separation and transfer at the CN/CuPc heterojunction.

\section{Conclusions}

In conclusion, $\mathrm{CN}$ nanosheet with high specific surface area were prepared through a two-step method. CuPc, a classic organic small molecular semiconductor possessing high mobility, outstanding visible-light absorption and physicochemical stability, was selected to construct CN/CuPc photocatalyst for PHE. Due to the broadened visible-light absorption and the enhancement of charge separation, the heterojunction exhibits a greatly improved PHE rate of up to 9.0 mmol g-1 $\mathrm{h}^{-1}$. The band position was determined and the photocatalytic mechanism of this organic heterojunction system was confirmed through various photoelectrochemical characterizations. This work demonstrates the prospect of high mobility organic semiconductors in photocatalytic reactions, providing a new strategy for the construction of efficient pure organic photocatalyst for PHE.

\section{Conflicts of interest}

There are no conflicts to declare.

\section{Acknowledgements}

The authors acknowledge the financial support from National Natural Science Foundation of China (52003143), the Natural Science Foundation of Shandong Province (ZR2019BB014) and the fund of China Scholarship Council (201908370037). Jianwu Sun acknowledges support by the Swedish Research Council (Vetenskapsrådet, grant no. 2018-04670), the Swedish Research Council for Environment, Agricultural Sciences and Spatial Planning (FORMAS, grant no. 2016-00559), and the Swedish Foundation for International Cooperation in Research and Higher Education (STINT, grant no. CH2016-6722).

\section{Notes and references}

1 J. Qi, W. Zhang, R. Cao, Adv. Energy Mater., 2018, 8, 1701620.

2 W. J. Ong, L. L. Tan, Y. H. Ng, S. T. Yong, S. P. Chai, Chem. Rev., 2016, 116, 7159-7329.

3 J. Wen, J. Xie, X. Chen, X. Li, Appl. Surf. Sci., 2017, 391, 72-123.

4 J. Fu, J. Yu, C. Jiang, B. Cheng, Adv. Energy Mater., 2018, 8, 1701503.

$5 \quad$ H. Huang, K. Xiao, N. Tian, F. Dong, T. Zhang, X. Du, Y. Zhang, J. Mater. Chem. A, 2017, 5, 17452-17463.

6 X. Sun, H. Huang, Q. Zhao, T. Ma, L. Wang, Adv. Funct. Mater., 2020, 30, 1910005. 
7 J. Chen, C. L. Dong, D. Zhao, Y. C. Huang, X. Wang, L. Samad, L. Dang, M. Shearer, S. Shen, L. Guo, Adv. Mater., 2017, 29, 1606198.

8 L. Ge, C. Han, J. Liu, J. Mater. Chem., 2012, 22, 11843-11850.

9 Y. Guo, S. Song, Y. Zheng, R. Li, T. Peng, Dalton Trans., 2016, 45, 14071-14079.

10 F. He, G. Chen, Y. Yu, S. Hao, Y. Zhou, Y. Zheng, ACS Appl. Mater. Interfaces, 2014, 6, 7171-7179.

11 Y. Sui, J. Liu, Y. Zhang, X. Tian, W. Chen, Nanoscale, 2013, 5, 9150-9155.

12 D. H. Wang, J. N. Pan, H. H. Li, J. J. Liu, Y. B. Wang, L. T. Kang, J. N. Yao, J. Mater. Chem. A, 2016, 4, 290-296.

13 J. Wang, Y. Zheng, T. Peng, J. Zhang, R. Li, ACS Sustain. Chem. Eng., 2017, 5, 7549-7556.

14 Z. Xing, Z. Chen, X. Zong, L. Wang, Chem. Commun., 2014, 50, 6762-6764.

15 H. Yan, Y. Huang, Chem. Commun., 2011, 47, 4168-4170.

16 F. Yu, Z. Wang, S. Zhang, H. Ye, K. Kong, X. Gong, J. Hua, H. Tian, Adv. Funct. Mater., 2018, 28, 1804512.

17 L. Yu, X. Zhang, C. Zhuang, L. Lin, R. Li, T. Peng, Phys. Chem. Chem. Phys., 2014, 16, 4106-4114.

18 X. Zhang, T. Peng, L. Yu, R. Li, Q. Li, Z. Li, ACS Catal., 2015, 5, 504-510.

19 X. Zhang, L. Yu, C. Zhuang, T. Peng, R. Li, X. Li, ACS Catal., 2014, 4, 162-170.

20 W. Zhou, T. Jia, H. Shi, D. Yu, W. Hong, X. Chen, J. Mater. Chem. A., 2019, 7, 303-311.

21 Z. Jiang, G. Dong, R. Wang, C. He, C. Wang, J. Sun, Sol. RRL, 2020, 4, 2000303.

22 Y. Shao, X. Xiao, Y. P. Zhu, T. Y. Ma, Angew. Chem. Int. Ed., 2019, 58, 14599-14604.

23 X. Sun, L. Shi, H. Huang, X. Song, T. Ma, Chem. Commun., 2020, 56, 11000-11013.

24 Z. Zhou, Y. Zhang, Y. Shen, S. Liu, Y. Zhang, Chem. Soc. Rev., 2018, 47, 2298-2321.

25 C. Wang, H. Dong, W. Hu, Y. Liu, D. Zhu, Chem. Rev., 2012, 112, 2208-2267.

26 Y. Lin, Y. Li, X. Zhan, Chem. Soc. Rev., 2012, 41, 4245-4272.

27 H. Wang, D. Yan, NPG Asia Mater. , 2010, 2, 69-78.

28 Y. Liu, L. Ma, C. Shen, X. Wang, X. Zhou, Z. Zhao, A. Xu, Chinese J. Catal., 2019, 40, 168-176.

29 P. Niu, L. Zhang, G. Liu, H.-M. Cheng, Adv. Funct. Mater., 2012, 22, 4763-4770.

30 J. T. Yin, Z. Li, Y. Cai, Q. F. Zhang, W. Chen, Chem. Commun., 2017, 53, 9430-9433.

31 P. Niu, G. Liu, H.-M. Cheng, J. Phys. Chem. C, 2012, 116, 1101311018. 